\title{
Treatment of Sepsis in pregnancy, Childbirth and the Puerperium: Update According to New Evidence
}

\section{Tratamiento de la Sepsis en el Embarazo, Parto y Puerperio, Actualización de Acuerdo a la Nueva Evidencia}

XVIII International Seminar on Health, Food and Human Nutrition

Corresponding Author:

Lino Arturo Rojas Pérez

linoarojas@hotmail.com

Published: 9 September 2021

Production and Hosting by

Knowledge $E$

(c) Lino Arturo Rojas Pérez et al. This article is distributed under the terms of the Creative Commons Attribution License, which permits unrestricted use and redistribution provided that the original author and source are credited.
Lino Arturo Rojas Pérez ${ }^{1,2}$, Lino Arturo Rojas Cruz², María Daniela Villagómez Vega $^{2}$, Augusto Ernesto Rojas Cruz ${ }^{2}$, and Andrés Eduardo Rojas Cruz ${ }^{2}$

${ }^{1}$ Facultad de Salud Pública, Carrera de Medicina, Facultad de Salud Pública, Escuela Superior Politécnica de Chimborazo, Riobamba, Ecuador 2ProSalud Medical Center, Jacinto Gonzales 1951 y Rey Cacha, Riobamba, Ecuador

\section{Abstract}

Introduction: Sepsis is a pathology that occurs due to a serious infectious process that can lead to death. It is characterized by organic and biochemical changes that determine an exaggerated response of the organism to infection. This infectious process can start anywhere in the body, subsequently causing bacteremia, systemic invasion through the blood, causing failure of multiple organs, and may even lead to the death of the person. Objective: The objective of this work is to describe the management of this pathology, according to the best evidence and above all updated. Methodology: Virtual libraries and scientific information search engines have been used to find the best available and most up-to-date evidence, and to meet the proposed inclusion criteria, in this research process, evidence and recommendations with a high level and scientific support in English or Spanish were used. of the last years. Results: 36 scientific articles were evaluated, such as clinical practice guides, systematic reviews, meta-analyzes, clinical trials, among others, which describes the most current management in a protocolized way. Discussion: Sepsis as a pathology increases maternal and perinatal morbidity and mortality and represents the third cause of maternal death worldwide and at the national level, knowing its proper management will help improve your prognosis and recovery. Conclusion: Since the complications of sepsis can produce multi-organ failure that will compromise maternal and perinatal health, knowledge of the updated management of this pathology is essential.

Keywords: sepsis, septic shock, infection.

\section{Resumen}

Introducción: La sepsis es una patología que se presenta por un proceso infeccioso grave que puede llevar a la muerte, se caracteriza por alteraciones orgánicas y bioquímicas que determinan una respuesta exagerada del organismo a la infección. Este proceso infeccioso puede iniciarse en cualquier sitio del cuerpo, provocar posteriormente bacteriemia, invasión sistémica a través de la sangre, causando falla de múltiples órganos, y pudiendo inclusive llevar al fallecimiento de la persona. Objetivo: El objetivo de este trabajo es describir el manejo de esta patología, de acuerdo a la mejor evidencia y sobre todo actualizada. Metodología: Se ha utilizado bibliotecas virtuales y buscadores de información científica para encontrar

Se ha ulizado biblotas vitules y buscadores de informán cientica para encontar 
la mejor evidencia disponible y más actualizada, y que cumplan los criterios de inclusión propuestos, en este proceso investigativo se utilizó evidencias y recomendaciones con un alto nivel y respaldo científico en idioma inglés o español de los últimos años. Resultados: Se evaluaron 36 artículos científicos como guías de práctica clínica, revisiones sistemáticas, metaanálisis, ensayos clínicos, entre otros, que describe en forma protocolizada el manejo más actual. Discusión: La sepsis como patología aumenta la morbimortalidad materna y perinata y representa la tercera causa de muerte materna a nivel mundial y a nivel nacional conocer su manejo adecuado ayudará a mejorar su pronóstico y su recuperación. Conclusiones: Debido que las complicaciones de la sepsis pueden producir falla multiorgánica que comprometerá la salud materna y perinatal, es fundamental el conocimiento del manejo actualizado de esta patología.

Palabras Clave: sepsis, choque séptico, infección.

\section{Introducción}

La sepsis es una complicación grave que se produce luego de un proceso infeccioso que puede iniciarse en cualquier sitio del organismo y que por vía sanguínea puede diseminarse a otros órganos pudiendo causar falla de los mismos y choque séptico que puede llevar a la muerte. Es una respuesta exagerada del organismo ante un proceso infeccioso.

Esta complicación médica pone en riesgo la vida de una persona, y en el embarazo sobre todo aumenta la morbimortalidad materna, perinatal y neonatal, por lo tanto, es fundamental el conocimiento más actual sobre su evaluación, diagnóstico y manejo debido que la sepsis es una de las principales causas de muerte en todo el mundo.

El objetivo de esta investigación es describir el manejo y sobre todo más actualizado de esta patología, de acuerdo a la mejor evidencia.

La pregunta que se utilizó para la búsqueda de la información en esta investigación es:

¿Cuál es el mejor tratamiento de la sepsis?

¿Cuál es el tratamiento más actualizado de la sepsis en el embarazo?

\section{Metodología}

Este trabajo de investigación científica se sustenta en la mejor evidencia disponible y actualizada del tratamiento de la sepsis en el embarazo, parto y puerperio, se tomó como base guías de práctica clínica y artículos científicos en idioma inglés o español de los últimos años.

Se realizó la búsqueda de la bibliografía en idioma inglés y español de los últimos cinco años en diferentes bibliotecas virtuales: Elsevier, Scopus, Cochane, BVS, ScienceDirect, así como buscadores de información científica: UptoDate, Mendeley, Web of Science, Google Schoolar, sobre sepsis y sepsis en el embarazo, utilizando 
como palabras clave: Sepsis, embarazo o pregnancy, management. Y de los resultados obtenidos se seleccionó la bibliografía científica de más alta calidad: Guías de práctica clínica, metaanálisis, revisiones bibliográficas, ensayos clínicos. Se ha seleccionado la bibliografía científica que presente los siguientes criterios de inclusión: estado del arte de los últimos cinco años (2015-2020), que presenten alta calidad de evidencia científica, que los autores no presenten conflictos de interés. Se ha descartado cualquier bibliografía científica que presentó cualquiera de estos criterios de exclusión: Estado del arte anterior a cinco años (<2015), que presenten baja calidad de evidencia científica. Se evaluaron 36 artículos científicos como revisiones sistemáticas, metaanálisis, guías de práctica clínica, ensayos clínicos, entre otros, todas con un fuerte nivel de evidencia.

\section{Resultados de búsqueda bibliográfica}

El equipo de trabajo encargado de esta investigación utilizó para la búsqueda de información plataformas virtuales como UpToDate, Pub-Med, the American College of Obstetricians and Gynecologists, Google Académico, entre otros, que permitieron encontrar artículos científicos y guías de práctica clínica en idioma inglés o español de los últimos años sobre sepsis, choque séptico infección materna y sobre todo durante el embarazo, parto o puerperio.

\section{Discusión}

La sepsis se presenta por un proceso infeccioso que desencadena una respuesta exagerada del organismo ante un proceso infeccioso, puede causar falla multiorgánica, choque séptico y comprometer la vida de una persona, se caracteriza por alteraciones orgánicas y bioquímicas que determinan un síndrome clínico que se caracteriza por una inflamación sistémica por infección [1-10].

Esta complicación médica pone en riesgo la vida de una persona, y es una de las principales causas de muerte en todo el mundo y la tercera causa de muerte materna a nivel mundial y a nivel nacional [11, 12].

De acuerdo a la nueva definición de sepsis consensuada en el 2016, llamada también Sepsis 3, la sepsis es una patología que se caracteriza por una respuesta exagerada del sistema inmunológico del organismo ante un proceso infeccioso, que produce disfunción o insuficiencia orgánica múltiple [10,13].

Cierto grupo de pacientes con sepsis pueden presentar choque séptico provocando anomalías circulatorias, metabólicas y celulares que aumentan la mortalidad. Que se caracteriza por hipotensión persistente y un lactato sérico superior a $2 \mathrm{mmol} / \mathrm{L}$ (18 $\mathrm{mg} / \mathrm{dL}$ ) a pesar de la reanimación adecuada con líquidos y que requiere el uso de vasopresores para mantener una presión arterial media igual o mayor de $65 \mathrm{~mm} \mathrm{Hg}$ $[4,14]$

Actualmente se eliminó al síndrome de respuesta inflamatoria sistémica (SIRS) como requisito para definir la sepsis, debido a que el mismo puede o no estar presente en 
procesos infecciosos, y además se eliminó la definición de sepsis grave. Lo que antes se llamaba sepsis grave ahora es la nueva definición de sepsis [4, 14].

La sepsis se inicia desde una infección en cualquier sitio del organismo, que puede complicarse con bacteriemia, posterior sepsis y choque séptico produciendo falla multiorgánica o síndrome de disfunción de órganos múltiples (MODS) y que puede terminar en la muerte [14] y si está embarazada aumenta la morbimortalidad materna, fetal y neonatal [15]. Pone en riesgo la vida de una persona y es la tercera causa de muerte materna a nivel mundial y a nivel nacional [11,12].

En el choque séptico se produce una falla circulatoria que amenaza la vida. Estos efectos de inicio pueden ser reversibles, pero rápidamente si no se trata adecuadamente pueden volverse irreversibles, lo que resulta en un fallo multiorgánico y la muerte [16].

La Sociedad de investigadores de Medicina Crítica Europeo y Norteamericano, conjuntamente con 19 sociedades internacionales especializadas en sepsis en el año 2016, describieron un puntaje para el probable diagnóstico de sepsis fuera de terapia intensiva, denominado quickSOFA (qSOFA) para facilitar la identificación de pacientes con alto riesgo de muerte por sepsis [5, 17-19]. Este puntaje es una versión modificada del puntaje de Evaluación Secuencial de Fallo de Órganos relacionado con Sepsis Ilamado SOFA [3].

El puntaje qSOFA tiene tres componentes, a cada uno se les asigna un punto [14, 20]:

- Frecuencia respiratoria (FR) igual o mayor de $22 / \min [3,20]$

- Presión arterial sistólica (PAS) igual o menor de $100 \mathrm{mmHg}[3,20]$

- Cualquier alteración del nivel de consciencia [3, 20]

Una puntuación igual o mayor de dos se asocia con probable sepsis fuera de la unidad de cuidados intensivos [3, 20].

En las mujeres embarazadas se produce una serie de cambios fisiológicos que modifican los valores considerados dentro de los parámetros normales fuera del embarazo como el aumento de un 30 al 50 por ciento de volumen plasmático y de un 20 al 30 por ciento de volumen celular [21], disminuye la resistencia vascular aproximadamente un 35 a un 40 por ciento y aumenta el gasto cardíaco [22]. La presión arterial sistólica disminuye aproximadamente 5 a $10 \mathrm{mmHg}$. durante el embarazo y un 15 por ciento de mujeres embarazadas tendrá una presión sistólica menor de $100 \mathrm{mmHg}$ dentro de un embarazo normal [15], por lo tanto, en el embarazo se recomienda diferente puntuación para determinar el quickSOFA, al que se le denomina quickSOFA obstétrico (omqSOFA), que es una modificación del qSOFA que se utiliza en personas no gestantes [11].

El puntaje omqSOFA tiene tres componentes, a cada uno se les asigna un punto [11]:

- PAS menor o igual a $90 \mathrm{mmHg}$ [11]

- FR mayor o igual de $25 / \mathrm{min}$ [11]

- Cualquier alteración del nivel de conciencia [11]

Una puntuación igual o mayor a dos, se asocia con probable sepsis materna fuera de la unidad de terapia intensiva [11]. 
Si se sospecha sepsis aplicando la puntuación omqSOFA se debe confirmar esta patología aplicando la puntuación llamada SOFA obstétrico (omSOFA) que es una variante del SOFA de personas no gestantes, que evalúa la disfunción orgánica [15]. Los parámetros que se evalúan en el omSOFA son los siguientes:

\section{Table 1}

SOFA obstétrico, tomado de Prevención, diagnóstico y tratamiento de la sepsis materna.

\begin{tabular}{|c|c|c|c|}
\hline Puntuación & 0 & 1 & 2 \\
\hline \multicolumn{4}{|l|}{$\begin{array}{l}\text { Variable SOFA } \\
\text { obstétrico }\end{array}$} \\
\hline $\begin{array}{l}\text { Respiración: } \\
\mathrm{PaO} 2 / \mathrm{FiO} 2\end{array}$ & $\geq 400$ & $300-400$ & $<300$ \\
\hline Plaquetas & $\geq 150.000 / \mathrm{mm}^{3}$ & $\begin{array}{l}100.000 \mathrm{a}< \\
150.000 / \mathrm{mm}^{3}\end{array}$ & $<100.000 / \mathrm{mm}^{3}$ \\
\hline Bilirrubinas (mg/dL) & $\leq 1,17$ & $1,17-1,87$ & $>1,87$ \\
\hline $\begin{array}{l}\text { Presión arterial media } \\
\text { (PAM) }\end{array}$ & $\geq 70 \mathrm{mmHg}$ & $<70 \mathrm{mmHg}$ & $\begin{array}{l}\text { Uso de vasoactivos para } \\
\text { mantener la PAM }\end{array}$ \\
\hline Nivel de consciencia & Despierto y Alerta & $\begin{array}{l}\text { Despierta al estímulo } \\
\text { verbal }\end{array}$ & $\begin{array}{l}\text { Despierta solo a estímulo } \\
\text { físico o dolor }\end{array}$ \\
\hline $\begin{array}{l}\text { Creatinina sérica } \\
(\mathrm{mg} / \mathrm{dL})\end{array}$ & $<1,0$ & $1,0-1,36$ & $>1,36$ \\
\hline
\end{tabular}

Guía de Evidencias y Recomendaciones: Guía de Práctica Clínica [23]. SOMANZ Guidelines for the Investigation and Management of Sepsis in Pregnancy [15].

Una puntuación de 2 o más es diagnóstico de sepsis obstétrica [11].

La escala de omSOFA es muy útil para el diagnóstico de sepsis en la mujer gestante y en el puerperio [11].

Las principales causa de sepsis en la mujer gestante se dividen en infecciones relacionadas directamente con el embarazo como corioamnionitis, endometritis, aborto séptico [24], infección abdominal de etiología obstétrica (ej. absceso pélvico); infecciones sin relación con el embarazo como pielonefritis, neumonía, fascitis, infección abdominal de etiología no obstétrica (ej. apendicitis complicada); infecciones nosocomiales como infección de vías urinarias debido a la colocación de sonda vesical, neumonía a consecuencia del uso de ventilador, infección de la herida quirúrgica [11].

La sepsis representa hasta el $28 \%$ de todas las muertes maternas, llegando a ser la tercera causa de muerte materna a nivel mundial y nacional. El diagnóstico y tratamiento oportuno y adecuado disminuye la morbilidad y la mortalidad materna y fetal [25].

Todas las pacientes con sepsis y choque séptico deben ser internadas en un hospital. Los pacientes con sepsis que responden a una terapia de reanimación adecuada de líquidos y no muestran signos evidentes de hipoperfusión tisular pueden ser ingresados en una unidad hospitalaria general, pero que cuente con personal preparado y capacitado para el manejo de este tipo de casos. Los pacientes que no responden al tratamiento inicial con líquidos y presenten una hipoperfusión tisular se diagnosticará de choque séptico y requieren el ingreso a una unidad de terapia intensiva $[26,27]$.

Las metas y principios básicos del manejo de la sepsis y el choque séptico incluyen $[1,26]$ : 
- Asegurar la vía aérea y corregir la hipoxemia.

- Establecer accesos venosos para la administración temprana de líquidos y antibióticos.

- Identificar y tratar la fuente de infección.

- Mantener una función adecuada del sistema de órganos, e interrumpir la progresión al síndrome de disfunción de múltiples órganos (MODS).

Asegurar la vía aérea. A todos los pacientes con sepsis se les debe suministrar oxígeno suplementario y la oxigenación debe controlarse con oximetría de pulso permanentemente. Cuando existe dificultad respiratoria o para la protección de las vías respiratorias es necesario proceder a la intubación y colocación de ventilación mecánica , debido que la encefalopatía hipóxica puede complicar la sepsis o un choque séptico, afectando el nivel de conciencia y agravar el cuadro clínico [1].

Establecer el acceso venoso. En pacientes con sepsis y choque séptico es prioritario el establecer un acceso venoso, en lo posible dos vías de gran calibre número 16 , tanto para la administración de líquidos para reanimación y para la administración de antibióticos. El acceso venoso periférico puede ser suficiente algunas veces, sobre todo para la reanimación inicial con líquidos, pero la mayoría necesitará un acceso venoso central en algún momento sobre todo para la administración de vasopresores. Sin embargo, la inserción de un catéter venoso central no debe retrasar la administración de líquidos para la reanimación y la administración de antibióticos. Se puede utilizar un catéter venoso central para infundir líquidos intravenosos, medicamentos (en particular vasopresores) y hemoderivados, así como para extraer sangre para estudios de laboratorio [26, 28].

Reanimación inicial con líquidos. El objetivo fundamental de la reanimación inicial es la rápida restauración de la perfusión tisular y la administración temprana de antibióticos [1].

La perfusión tisular se logra fundamentalmente mediante la administración de líquidos intravenosos a dosis de $30 \mathrm{~mL} / \mathrm{kg}$ peso, dentro de las primeras tres horas después de la presentación del cuadro clínico, usualmente se administra cristaloides balanceados como el Lactato de Ringer o solución salina normal [1, 11, 26]. La terapia con líquidos se debe administrar en bolos de infusión rápida de $500 \mathrm{~mL}$ hasta llegar a la dosis total recomendada de $30 \mathrm{~mL} / \mathrm{kg}$ de peso. La respuesta clínica y hemodinámica y la presencia o ausencia de edema pulmonar deben evaluarse antes y después de cada bolo [1]. Si luego de la dosis total de líquidos persiste la hipotensión o la hipoperfusión tisular, significa que la paciente ya no necesita más líquidos, sino más bien se recomienda en ese caso el uso de vasopresores [1, 11].

En todos los pacientes con sepsis o choque séptico se debe colocar una sonda vesical permanente para vigilar la diuresis horaria que es uno de los marcadores de la perfusión, función renal y del gasto cardiaco; la diuresis normal debe ser igual o mayor de $0,5 \mathrm{~mL} / \mathrm{kg}$ de peso/hr [26].

Tratamiento antibiótico. El tratamiento antibiótico debe administrarse para los organismos sospechosos de la infección y responsables de la sepsis, en lo posible dentro de la primera hora de presentación del cuadro clínico [1]. Cuando la administración del 
antibiótico se retrasa más de una hora, aumenta la morbimortalidad [11]. Por lo tanto, se debe iniciar empíricamente el tratamiento antibiótico por vía intravenosa, siempre orientado en la probable causa del proceso infeccioso dentro de la primera hora de su diagnóstico [11, 12]. Por cada hora de retraso del uso del antibiótico la mortalidad materna aumenta en un ocho por ciento [15].

Se recomienda antes de la administración de los antibióticos tomar muestras para cultivos, pero que esto no retrase el inicio de los mismos [11].

Dentro de las bacterias principales responsables de la sepsis en obstetricia están el Streptococcus del grupo A (pyogenes), Escherichia coli y Staphylococcus aureus $[29,30]$.

Existe varios esquemas que se pueden utilizar en casos de sepsis y choque séptico en la mujer gestante o puérpera, dentro de los principales:

- Penicilina intravenoso 5 '000.000 Ul administradas cada cuatro horas, asociado a la administración intravenosa de Gentamicina a dosis de $5 \mathrm{mg} / \mathrm{kg}$ cada veinte y cuatro horas, asociado a Metronidazol intravenoso $500 \mathrm{mg}$ cada ocho horas $[12,31]$.

- Ampicilina/sulbactam intravenoso $3 \mathrm{~g}$ administrados cada seis horas, asociado a la administración intravenosa de Gentamicina a dosis de $5 \mathrm{mg} / \mathrm{kg}$ cada veinte y cuatro horas, asociado a Clindamicina intravenoso $900 \mathrm{mg}$ cada ocho horas $[12,31]$.

- Ceftriaxona intravenoso $1 \mathrm{~g}$ administrado cada doce horas, asociado a Metronidazol intravenoso 500 mg cada ocho horas o Clindamicina intravenoso 900 mg cada seis horas [12, 31].

- Piperacilina/tazobactam intravenoso administrada 4,5 g cada seis horas [12].

La duración del tratamiento antibiótico debe ser de 7 a 10 días [11]. Pero en pacientes neutropénicos, el tratamiento debe continuar hasta que la neutropenia se haya resuelto o el curso de antibióticos planeado esté completo, lo que sea más prolongado [1].

Se recomienda realizar exámenes de laboratorio dentro de los 45 min de la presentación del cuadro clínico, pero estos no deben retrasar la administración de líquidos y antibióticos:

Biometría hemática completa, coagulograma, dímero D, química sanguínea, pruebas de función renal y hepática. El análisis de estos estudios pueden ayudar a respaldar el diagnóstico, indicar la gravedad de la sepsis y servir de ayuda para seguir la respuesta terapéutica [1].

Lactato sérico: si es mayor de $2 \mathrm{mmol} / \mathrm{L}$ indica una hipoperfusión tisular y agravamiento de su cuadro séptico, es de mucha ayuda para seguir la respuesta terapéutica [32, 33].

Gasometría arterial: Pueden revelar acidosis, hipoxemia o hipercapnia [1].

Hemocultivos tanto para aeróbicos y anaeróbicos, de al menos dos sitios diferentes, urocultivo y cultivos de fuentes sospechosas (por ejemplo, esputo, catéter intravascular, fluidos corporales entre otros). Si tiene un catéter vascular central que se sospecha que 
es la fuente de infección, la sangre debe obtenerse tanto del catéter como de los sitios periféricos [1].

Estudios radiológicos dirigidos al sitio sospechoso de infección como Rx de tórax, tomografía axial computarizada de tórax, abdomen, entre otros [1].

Procalcitonina: Su determinación no tiene suficiente evidencia en el diagnóstico y manejo de la sepsis, su uso está justificado en el seguimiento de pacientes con neumonía adquirida en la comunidad para el desescalado antibiótico [1]. El uso de procalcitonina para guiar la desescalada antimicrobiana en sepsis no produjo ningún beneficio para disminuir la mortalidad [34].

Si se presenta acidosis metabólica se sugiere el manejo con bicarbonato [1].

Luego del manejo inicial con líquidos y el uso de antibióticos empíricos se debe investigar e identificar y controlar la fuente de infección, debido que si no se elimina la misma los antibióticos solos no ayudarán a la mejoría clínica [33].

Si luego de la reanimación con líquidos persiste la hipotensión o si desarrolla edema pulmonar cardiogénico, se debe iniciar vasopresores intravenosos [1] y la norepinefrina es el de primera elección [1, 11].

Si a pesar del uso de líquidos a dosis adecuadas y el uso de vasopresores, persiste la hipotensión, se recomienda utilizar glucocorticoides [1]. El corticoide que se recomienda en este caso es la hidrocortisona 100 mg por vía intravenosa cada 8 hr por cinco a siete días [35].

Tromboprofilaxis. Tanto el embarazo y la sepsis son factores de riesgo para el tromboembolismo venoso, por lo tanto [15], se recomienda tromboprofilaxis mecánica y si no existe contraindicación, también tromboprofilaxis farmacológica, y como medicamento de primera elección la heparina de bajo peso molecular [11].

Identificación y control de la fuente de infección. Se recomienda identificación y control de la fuente de infección dentro de las primeras seis a doce horas [1].

Si se presenta sepsis de origen intrauterino independientemente de la edad gestacional se debe considerar la interrupción del embarazo. Se sospecha en casos de fiebre materna asociada a secreción purulenta endocervical, antecedente de ruptura prematura de membranas, taquicardia fetal o materna, o presencia de hipersensibilidad uterina $[11,15]$. $Y$ en casos de embarazos pretérmino que se va a interrumpir entre las 24 a 36 semanas con 6 días se debe administrar corticoides para maduración pulmonar y disminuir el riesgo de síndrome de membrana hialina, hemorragia intraventricular y enterocolitis necrotizante [11,36]. La sepsis no es una contraindicación para el uso de corticoides [15].

Si la sepsis se presenta en embarazos pretérmino y si el origen es extrauterino, se puede tratar la infección y si esto se consigue se debe continuar con el embarazo [11], y en embarazos atérmino es razonable considerar el parto como un medio para mejorar los esfuerzos de reanimación materna [15].

Si se presenta hiperglicemia igual o mayor de $180 \mathrm{mg} / \mathrm{dL}$, se recomienda la administración de insulina [11].

Uno de los objetivos de la respuesta clínica adecuada del manejo de la sepsis y choque séptico es a través de objetivos clínicos, incluida una presión arterial media 
(MAP) igual o mayor de $65 \mathrm{mmHg}$, y una diuresis igual o mayor de $0,5 \mathrm{~mL} / \mathrm{kg}$ de peso/hr [1].

\section{Conclusiones}

La sepsis y el choque séptico en mujeres embarazadas o en el puerperio aumentan la morbimortalidad tanto materna como perinatal por lo tanto es fundamental su prevención, diagnóstico oportuno y el manejo adecuado de acuerdo a la mejor evidencia. Los puntos básicos para el manejo de esta patología incluyen:

- Asegurar la vía aérea

- Establecer un acceso venoso

- Administración de líquidos dentro de las tres primeras horas

- Administración de antibióticos dentro de la primera hora

- Si luego de la administración de líquidos persiste la hipotensión o la hipoperfusión tisular, se debe utilizar vasopresores

- Si luego del uso de líquidos y vasopresores persiste la hipotensión o la hipoperfusión tisular, administrar glucocorticoides

- Tratamiento de la acidosis

- Control de la hiperglicemia

- Realizar exámenes de laboratorio pertinentes

- Identificación y control de la fuente de infección dentro de las primeras seis a doce horas

- Tromboprofilaxis

- Decisión de continuar o no el embarazo de acuerdo al caso

\section{Agradecimientos}

Agradecemos a la Facultad de Salud Pública y en especial a la Carrara de Medicina por organizar este congreso médico que aporta científicamente para la actualización de conocimientos tanto de los estudiantes y de los docentes y por permitirnos publicar esta revisión científica que servirá como fuente de información y consulta para los profesionales de la salud.

En este trabajo de investigación se realizó una revisión no sistemática bibliográfica.

\section{Conflicto de Intereses}

No existen de parte de ninguno de los autores. 


\section{Limitación de Responsabilidad}

Las opiniones y recomendaciones expresadas en este artículo científico son responsabilidad de los autores y de ninguna manera de la institución en la que trabajamos.

\section{Fuentes de Apoyo}

Propia de los autores.

\section{References}

[1] Schmidt G, Mandel J. Evaluation and management of suspected sepsis and septic shock in adults. UpToDate. 2019. Available from: https://www.uptodate.com/contents/ evaluation-and-management-of-suspected-sepsis-and-septic-shock-in-adults/print?search= sepsis\&source=search_result\&selectedTitle=2 150\&usage_type=default\&display_rank=2

[2] Tang Y, Choi J, Kim D, et al. Clinical predictors of adverse outcome in severe sepsis patients with lactate 2-4 mM admitted to the hospital. QJM. 2015;108(4):279-87.

[3] Neviere R. Sepsis syndromes in adults: Epidemiology, definitions, clinical presentation, diagnosis, and prognosis. UpToDate. 2018. Available from: https://www.uptodate.com/contents/ sepsis-syndromes-in-adults-epidemiology-definitions-clinical-presentation-diagnosis-and-prognosis/ print?search=sepsis\&source=search_result\&selectedTitle=1\$\{|sim\}\$150\&usage_type= default\&display_rank=1

[4] Kalil A. Septic Shock. Practice Essentials. Medscape. 2018. Available from: https://emedicine.medscape. com/article/168402-overview\#showall

[5] Rudd KE, Seymour CW, Aluisio AR, et al. Association of the quick sequential (sepsis-related) organ failure assessment (qsofa) score with excess hospital mortality in adults with suspected infection in low- and middle-income countries. JAMA. 2018;319(21):2202.

[6] Lamontagne F, Rochwerg B, Lytvyn L, et al. Corticosteroid therapy for sepsis: A clinical practice guideline. BMJ. 2018;362:3284.

[7] Kall A. Septic shock clinical presentation: History, physical examination, complications. Medscape. 2019. Available from: https://emedicine.medscape.com/article/168402-clinical\#showall

[8] Kall A. Septic shock differential diagnoses. Medscape. 2019. Available from: https://emedicine. medscape.com/article/168402-differential

[9] Fang F, Zhang Y, Tang J, et al. Association of corticosteroid treatment with outcomes in adult patients with sepsis. JAMA Intern Med. 2019;179(2):213.

[10] Bokhari AM. Bacterial Sepsis: Practice Essentials, Background, Etiology. Medscape. 2019. Available from: https://emedicine.medscape.com/article/234587-overview\#showall

[11] Arroyo K, Carreño M, Ocampo J. Prevención, diagnóstico y tratamiento de la sepsis materna. Guía de evidencias y recomendaciones: Guía de práctica clínica. Vol. 1. CENETEC. México: CENETEC; 2018. 1-41 p. Available from: http://www.cenetec-difusion.com/CMGPC/GPC-IMSS-272-18/ER.pdf

[12] Calle J, Ríos P, Yuen Chon V, Rojas L, Rivadeneira J, Pareja C. Score MAMÁ y claves obstétricas. Protocolo. Primera Ed. Quito: Gerencia Institucional de Implementación de Disminución Mortalidad Materna. Ministerio de Salud Pública del Ecuador; 2017.

[13] WHO. Statement on Maternal Sepsis. World Health Organisation; 2017. Available from: www.who. int/reproductivehealth

[14] Kall A. Septic shock: Practice essentials, background, pathophysiology. Medscape. 2019. Available from: https://emedicine.medscape.com/article/168402-overview\#showall

[15] Bowyer L, Robinson H, Angela M, et al. SOMANZ Guidelines for the investigation and management of sepsis in pregnancy. 2017. Available from: https://www.somanz.org/downloads/2017SepsisGuidelines. pdf

[16] Gaieski D, Mikkelsen M. Definition, classification, etiology, and pathophysiology of shock in adults. UpToDate. 2018. Available from: https://www.uptodate.com/contents/ definition-classification-etiology-and-pathophysiology-of-shock-in-adults/print?search= choque\&source=search_result\&selectedTitle=1\$\{|sim\}\$150\&usage_type=default\&display_rank=1

[17] Shankar-Hari M, Phillips GS, Levy ML, et al. Developing a new definition and assessing new clinical criteria for septic shock: Third international consensus definitions for sepsis and septic shock (Sepsis-3). JAMA. 2016;315(8):775. 
[18] Singer M, Deutschman CS, Seymour CW, et al. The third international consensus definitions for sepsisand septic shock (Sepsis-3). JAMA. 2016;315(8):801-10.

[19] Seymour CW, Liu VX, Iwashyna TJ, et al. Assessment of clinical criteria for sepsis: For the third international consensus definitions for sepsis and septic shock (Sepsis-3). JAMA. 2016;315(8):762-74.

[20] Koyama SI, Yamaguchi Y, Gibo K, Nakayama I, Ueda S. Use of prehospital qSOFA in predicting inhospital mortality in patients with suspected infection: A retrospective cohort study. PLoS One. 2019 [cited 2019 May 12]; Available from: https://www.ncbi.nlm.nih.gov/pmc/articles/PMC6504075/pdf/pone. 0216560.pdf

[21] Bauer K. Maternal adaptations to pregnancy: Hematologic changes. UpToDate. 2018. Available from: https://www.uptodate.com/contents/maternal-adaptations-to-pregnancy-hematologic-changes/print? sectionName=PLASMAVOLUME\&search=cambiosfisiologicosenelembarazo\&topicRef=443\&anchor= H1549271045\&source=see_link

[22] Foley M. Maternal adaptations to pregnancy: Cardiovascular and hemodynamic changes. UpToDate. 2017. Available from: https://www.uptodate.com/contents/ maternal-adaptations-to-pregnancy-cardiovascular-and-hemodynamic-changes/print?search= cambiosfisiologicosenelembarazo\&source=search_result\&selectedTitle=1\$\{lsim $\} \$ 150 \&$ usage_type= default\&display_rank=1

[23] Prevención, Diagnóstico y Tratamiento de la sepsis materna. Guía de referencia rápida: Guía de Práctica Clínica. 2018. Available from: http://www.cenetec-difusion.com/CMGPC/GPC-IMSS-272-18/RR.pdf

[24] Morgan J, Roberts S. Maternal Sepsis. Obstet Gynecol Clin North Am. 2013;40(1):69-87.

[25] Albright CM, Mehta ND, Rouse DJ, Hughes BL. Sepsis in Pregnancy: Identification and Management. J Perinat Neonatal Nurs. 2016;30(2):95-105. Available from: https://www.ncbi.nlm.nih.gov/pubmed/ 26825620

[26] Kall A. Septic shock treatment \& management: Approach considerations, general treatment guidelines in septic shock, goals of hemodynamic support. Medscape. 2019. Available from: https://emedicine. medscape.com/article/168402-treatment\#showall

[27] Clardy P, Reardon C. Critical illness during pregnancy and the peripartum period. UpToDate. 2019. Available from: https://www.uptodate.com/contents/ critical-illness-during-pregnancy-and-the-peripartum-period/print?search=sepsis\&topicRef= $1613 \&$ source=see_link

[28] Schmidt GA, Mandel J. Evaluation and management of suspected sepsis and septic shock in adults. UpToDate. 2018. Available from: https://www.uptodate.com/contents/ evaluation-and-management-of-suspected-sepsis-and-septic-shock-in-adults/print?search= sepsis\&source=search_result\&selectedTitle=2 ${ }^{\sim} 150 \&$ usage_type=default\&display_rank $=2$

[29] Cordioli R, Cordioli E, Negrini R, Silva E. Sepsis and pregnancy: Do we know how to treat this situation? Rev Bras Ter Intensiva. 2013.

[30] Spera AM, Bianco V, Simeone D, Viceconte G, Tosone G, Orlando R. Sepsis in pregnant and puerperal women living in high income countries: An update for clinicians. J Gynecol Obstet. 2017.

[31] Tita A. Intra-amniotic infection (clinical chorioamnionitis or triple I). UpToDate. 2019. Available from: https://www.uptodate.com/contents/intra-amniotic-infection-clinical-chorioamnionitis-or-triple-i/print? sectionName=Antibiotictherapy\&search=gentamicina\&topicRef=475\&anchor=H15\&source=see_link

[32] Howell MD, Davis AM. Management of sepsis and septic shock. JAMA. 2017;317(8):847-8.

[33] Rhodes A, Evans LE, Alhazzani W, et al. Surviving sepsis campaign: International guidelines for management of sepsis and septic shock: 2016. Intensive Care Med. 2017;43:304-77.

[34] Andriolo BN, Andriolo RB, Salomão R, Atallah ÁN. Effectiveness and safety of procalcitonin evaluation for reducing mortality in adults with sepsis, severe sepsis or septic shock. Cochrane database Syst Rev. 2017;1(1):CD010959.

[35] Kaufman D, Mancebo J. Glucocorticoid therapy in septic shock. UpToDate. 2018. Available from: https://www.uptodate.com/contents/glucocorticoid-therapy-in-septic-shock/print?search= sepsis\&topicRef=1613\&source=see_link

[36] Committee on Obstetric Practice. Antenatal corticosteroid therapy for fetal maturation. Acog Committee Opinion. Am Coll Obstet Gynecol. 2017;102-9. 